\title{
Progress on the Modern Nursing of Pneumonia Pathogenic Bacteria of Ventilator-associated Pneumonia
}

\author{
Yanping $\mathbf{L i}^{*}$
}

The People’s Hospital of Langzhong City, Langzhong, Sichuan 637400, China

\begin{abstract}
The mortality of newborns due to pulmonary infection remains high, which raises a problem for modern nursing management. With the emerging and gradual maturity of the newborn major, the wide application of ventilator for newborns in the intensive care unit (ICU) becomes the important factor in the successful salvage of high-risk newborns. This has presented a problem, however, due to misoperation and other factors. This paper starts from the angles of diagnosis criteria and morbidity of Ventilator-associated Pneumonia (VAP) to analyse the pathogenic bacteria of VAP and the progress, to provide some thoughts for the development trend of modern nursing of
\end{abstract} VAP.

\section{KEYWORDS}

Newborn

Pneumonia

Ventilator association

Pathogenic bacteria

Modern nursing

\section{Introduction}

Mechanical ventilation of ventilator is a kind of effective and important measure for the salvage of critical newborns, is widely applied in neonatal intensive-care unit (NICU) and achieves good effects improving obviously the survival rate of newborns [1]. However, in the actual treatment of mechanical ventilation, misoperation or other factors often cause VAP, seriously threatening the safety and lives of the newborns.

Ventilator-associated Pneumonia refers to pneumonia occurring 48 hours after mechanical ventilation of the ventilator is unplugged, an important type of Hospitalacquired Pneumonia (HAP). The pneumonia occurring within four days after mechanical ventilation is called early-onset VAP, and the pneumonia occurring five days after mechanical ventilation is called late-onset VAP. Ventilatorassociated Pneumonia, in clinical terms, is called clinically refractory pneumonia and seriously threatens the lives of newborns. This paper analyze the diagnosis of criteria, morbidity, etiology and high-risk factors of VAP by retrospective research to put forward the nursing measures of

\section{Copyright ( 2016 Yanping Li}

doi: $10.18686 /$ jn.v5i4.8

Received: August 21, 2016; Accepted: November 2, 2016; Published on-line: December 29, 2016

This is an open-access article distributed under the terms of the Creative Commons Attribution Unported License (http://creativecommons.org/ licenses/by-nc/4.0/), which permits unrestricted use, distribution, and reproduction in any medium, provided the original work is properly cited.

${ }^{*}$ Corresponding author: The People's Hospital of Langzhong City, Langzhong, Sichuan 637400, China. E-mail: drlyp400@163.com prevention.

\section{VAP diagnosis of newborns}

Other than for clinical situations, laboratory data, imageology and etiology materials, there has not been a uniform diagnosis criteria for VAP yet. Currently, the combination of microbiological examination of lung tissues and pathological diagnosis is the most reasonable diagnosis method in the medical fraternity. The combination of etiological examination and clinical and X-ray manifestations can improve the accuracy of VAP diagnosis. With the wide application and in-depth research of bronchofiberscope sampling technology, the accuracy of VAP diagnosis further improves [1].

Domestic and foreign literature reports that G-bacillus has the biggest proportion [2] in infectious bacteria of VAP, and infectious bacteria of VAP are mostly conditioned pathogen, mainly including Pseudomonas aeruginosa, Klebsiella pneumonia, Acinetobacter and Escherichia coli. Some patients are co-infected by many pathogenic microorganisms. With the wide application of the thirdgeneration cephalosporin, in recent years, the infection of extended spectrum beta-lactamase (ESBL)s-produced bacteria is gradually valued in NICU.

\section{Progress on the nursing of VAP of newborns 3.1. Strengthening the education of infection control for medical personnel}

The experimental research of one hospital finds that after the education of prevention knowledge relating to VAP for medical personnel in ICU, the occurrence of VAP low- 
ers more than $50 \%$. It shows that by strengthening knowledge training of medical personnel, a significant degree in lowering VAP occurrence can be secured. Thus, medical institutions at all levels must value highly infection control education for medical personnel.

\subsection{Enforce antiseptic hand washing}

The main purpose of washing hands is to lower infection rate in the process. Therefore, before and after nursing operations, especially in the process of mechanical ventilation, antiseptic hand washing must be strictly conducted after touched respiratory secretions every time. Besides, disposable glove can be worn in respiratory nursing, but hands must be washed carefully before and after the wearing of gloves.

\subsection{Strengthening environment management}

Air cleaning systems must be installed in wards, and disinfectant agent must be used when mopping the floor. Regularly sterilize the air with ultraviolet ray. Strictly limit visitation hours. Medical personnel and family members must wear masks, cups and isolation gowns. And when necessary, take protective measures for high-risk people.

\subsection{Strengthening the relevant nursing of ventilator 3.4.1. Cutting other infection links}

Strengthening the education of infection control for medical personnel can effectively reduce the occurrence of VAP.

\subsubsection{Keeping the air in wards clean}

Open windows for ventilation twice every day, and use chlorine-containing disinfectant solution to wipe or mop object plane and floor twice every day; regularly use Kengewang air disinfecting machine to sterilize air within 24 hours every day; limit the traffic of personnel and maintain air quality.

\subsubsection{Position}

Semi-reclining position can reduce the backflow of gastric content and lower the occurrence of VAP. Therefore, semi-reclining position for infants is an effective method. Centers for Disease Control and Prevention suggest, the bedside to be raised by 30-35 degrees when conducting mechanical ventilation for patients.

\subsubsection{Strengthening the management of the pipes of ventilator and humidification equipment}

By regularly replacing the pipes of the ventilator, bacteria contamination in the pipes can be reduced, which is an important step in lowering VAP [3]. However, replacing of the pipes frequently will also increase the opportunity of bacterial infection, so should only replace them every three or four days. Water collection bottle should place on the lowest position on respiratory circle and regularly pour the collected water in the water collection bottle. Sterile water for injection should pour in atomizer and humidification bottle and replace them regularly. Sterilize humidification bottles should replace every week; the liquid in the pipes of sputum aspirator and wide-mouth bottle substitute in every shift, and clean and sterilize the pipes of sputum aspirator and wide-mouth bottle.

\subsection{The nursing of respiratory tract}

There is a gap between glottis and airbag of intubation patients where pars pharyngoorale secretions are easily produced and stored, which is an important source [4] of pathogenic bacteria of VAP because the secretions containing high-concentration bacteria can be mistakenly sucked into the respiratory tract. Therefore, fully draining the agglomerates over and below the gasbag can prevent VAP and the VAP caused by primary endogenous bacteria can be prevented by suction under glottis. The specific method is to suck the secretions in respiratory tract, mouth and nose successively in sterile operation. The time of every sputum suction is less than $15 \mathrm{~s}$ and a transitory suction of oxygen should be offered before sputum suction; besides, physical treatment can also help patients improve the clearing ability of their respiratory tract, such as turning over and percussing breast and back, which is generally conducted gently twice every two hours. But for premature infants, percussing the back and other violent methods should not be used.

\subsection{The care of mouth cavity}

The principle of respiratory tract causing VAP is: the mouth cavity is the initial part of the respiratory tract. After the mucosal barrier between pars pharyngoorale and lower respiratory tract is damaged by intubation, the pathogenic bacteria in pars pharyngoorale moves to lower respiratory tract causing VAP. Therefore, strengthening oral nursing can effectively lower the occurrence of VAP of newborns. Different oral care methods have different effects. According to the experimental analysis of one hospital [5], after oral nursing, the number of pathogenic bacteria in control group decreased $16.7 \%$ while that in experimental group decreased $47.5 \%$. The VAP occurrence of experimental group (21\%) is lower obviously than that of the control group (50\%), which shows that the combination of oral swab method and oral rinses method can better lower VAP occurrence than conventional swab method.

\subsection{Replacing invasive ventilation with noninvasive ventilation}

For newborns, we can adopt non-invasive technologies, such as continuous positive airway pressure (CPAP) mask, nasal congestion CPAP and pharyngeal synchronized intermittent mandatory ventilation (SIMV) technology, which avoid infection accompanied by incubation of respiratory tract. At the same, these can treat primary diseases and prevent VAP brought on by invasive ventilation. But 
for some high-risk cases, especially those whose massive secretions cannot be discharged effectively and those who have a choking hazard because their respiratory tract cannot be protected effectively, non-invasive ventilation is not recommended.

\subsection{Improving the immunity of body}

Adopting increasing nutrition and supporting treatment to ensure the body has enough heat to improve immunity can help prevent VAP from its root cause. Adopt gamma globulin, hemalbumin and plasma for premature infants and critical infection patients according to situations to improve the resistance ability of the body. Experimental research [3] shows that when adopting a large dose of gamma globulin treatment, the infection rate of VAP is $42.1 \%$ while the infection rate of VAP for some infants is $100 \%$, which shows gamma globulin has prevention and synergy therapy functions for VAP, a key point to nursing.

\subsection{Assisting sputum excretion}

Help infants turn over, percuss their backs every two hours and use mask and hand force to percuss their backs from down to up to assist sputum excretion. But this does not apply to premature infants. Other than routinely turning on the heating humidifier, $1 \mathrm{ml}$ of normal saline can be dropped into the endotracheal tube of the infants with ropy sputum to dilute sputum after sputum excretion every time.

\subsection{Reasonably using antibiotics}

Conduct bacterial cultivation in the sputum of pars oralis pharynx and lower respiratory tract of the mechanical ventilation infants. Choose highly targeted antibiotics according to drug sensitivity results, avoid medication according to experience and reduce unnecessary preventive medication.

Correct sputum suction; do not suck for sputum within 30 minutes after meals, and strictly obey sterile technologies operation. Raise sputum aspirator lightly when rotating it rightwards or leftwards and when sucking sputum. The sputum suction time is $<15$ seconds every time. Suck all secretions in air passage first, then suck all secretions in oral and nasal cavity after replacing pipe. Offer two minutes of pure oxygen inhalation before sputum suction.

We should adopt preventive measures for infants, who are receiving mechanical ventilation treatment, with VAP as a kind of hospital-acquired disease. The younger the gestational age, the lower the birth weight and the higher the occurrence of VAP; the longer the duration of mechanical ventilation, the more easily VAP occurs. Thus, we should emphasize infants with young gestational age and long mechanical ventilation in the prevention and treatment of VAP. The longest mechanical ventilation time in the group of infants should be 14 days. As the time of mechanical ventilation extends, the occurrence of VAP increases. However, we should strengthen the management of respiratory tracts and ventilator and duly for the replacement for pipes of the ventilator, but the frequent replacing of pipes of ventilators can also increase infection opportunities. It was reported that there is no obvious difference in the types of bacteria and the number of bacterial colonies on the pipes replaced every two days and the pipes replaced every seven days. Our hospital replaces the pipes of ventilator every 72 hours. We should strengthen the nursing of all infants receiving mechanical ventilation treatment. The currently recognised effective prevention measures include regular position change, avoiding aspiration, $45^{\circ}$ semi-reclining position, paying attention to the sterile operation in sputum suction and hand hygiene, reasonably using antibiotics and keeping oral cavity clean.

\section{Conclusion}

Ventilator-associated Pneumonia is a kind clinically common infection situation. It requires clinical strengthening of infection control, education of nursing personnel, accelerating the relevant management of the ventilator, mouth care, the application of noninvasive ventilation technology, and improving fundamentally the immunity of patients. I believe that with the gradual development of medical technologies, the occurrence of VAP infection will gradually decrease under the driving of technologies, with nursing levels further developing.

\section{References}

1. Duan H, Wang H. The clinical analysis of VAP. Sichuan Medical Journal. 2009;(3):11-14.

2. Song $X$, Tang $X$, Chen $H$. The status quo of VAP and its nursing research. Journal of Qilu Nursing. 2009; (23):23-24.

3. Li Y, Huang $\mathrm{H}$, Wang $\mathrm{W}$. The bacteria and drug sensitivity analysis of VAP. Modern Chinese Doctor. 2009;(17):17-19.

4. Dong J. 76 cases of clinical analysis of VAP. Chinese Journal of Coal Industry Medicine. 2010;(02):12-18.

5. Teng G. The overview and nursing of pathogenic bacteria of VAP. Lectures and Summary. 2010;(23):23-24. 